\title{
Comparative Study of Global Indicators of Core Competence of Nurses*
}

\author{
Si CHEN, Jihong WANG* \\ Department of Nurse, Beihua University, Jilin, China
}

\begin{abstract}
Based on domestic and overseas nurses core difference in scale for each dimension can be compared through their national conditions and cultural factors to identify suitable, can be indicators of nursing core competencies for the future development and use for reference.
\end{abstract}

Index Terms - nurse, core competence, education.5.

\section{Introduction}

Back in the late 1970s, English education would put forward the concept of core competence, it is emphasized that the occupation when the change occurred, or when changes in labor organization, the workers still possess the ability to work [1].

The term specialist was first applied in nursing in the early years of the 20th century as more postgraduate courses in specific areas of practice became available [2]. Nursing core Competency (NCC) is the core competence of this particular nursing profession is engaged in clinical nursing work every caregiver must possess basic comprehensive ability[3].Nursing scholars in various countries of the proposal initiated by the 2003 International Council of Nurses (ICN) has developed core competencies of nurses in line with national realities framework, allowing countries to develop the capacity of nurses evidence-based standards [4].

Review of the literature collected by the United States, Britain, Canada, China, Norway and other countries in recent years more authoritative core competency scale, through expert analysis, the removal of national cultural backgrounds influence their ability to compare differences in the ability to extract the core countries of nurses Advanced advantage.

This document may be used by nurse practitioners to support their self-refection, self-evaluation and continuing competence, as well as to educate others about their role [4]. Nurse practitioner educators may use it for curriculum development. And it is a useful resource for helping others including government agencies, employers, health providers and the public - to understand what they can expect of nurse practitioners [5].

\section{The Current Situation}

Recently the American Nurses core competency scales are set by Maine nursing education and practice team, scale using Bloom (1956) the knowledge, skills, attitudes. From three core competencies elaborated 11 nurses, including 11 core competencies include: professional, leadership, patient centered care, evidence-based practice, teamwork, communication, system practices, information and technology, safety, quality improvement, geriatrics;

In the light of current political and professional debate in the United Kingdom concerning preparing nurses for competence, this paper takes an historical perspective, and considers how nursing competence was Derned historically through an analysis of statutory syllabuses and nursing textbooks $1874 \pm 1977$ [6]. Competence was perceived by nursing textbook writers to have four facets. Firstly, it involved the moral character of the nurse; secondly, it required technical knowledge, practical skill and procedure; thirdly, it depended on the role of the ward sister; and fourthly, it relied on the professional etiquette of right relationships [7].

Maine's nursing team found that the current accelerated aging of the US population, aged care should also be one of the current nursing staff must have the ability to carry out modifications on the basis of the 2010 US [8]core competencies framework, finalize join Geriatrics knowledge in the nursing core competency framework. Meanwhile, the United States and Norway care experts agree that the core competence of nurses should be rated at different stages of the framework, the United States will be divided into five stages nurses are: novice, beginner, director (independent lowest level), skilled experts [9].

Norwegian nurses stressed that due to personal experience and personal differences, nursing stage is not defined by time, no exact standard (three nurse care $=$ ) [10]. For the assessment phase of the nursing staff, Chinese nursing assessment theory combined with practical application of working age to assess the stage of nurses [11]. Norwegian specialist nurses think you should re-evaluate the level of nurses to assess the ability of nurses. OKLAHOMA US states for nurses to assess the ability of nurses educated in accordance with the classification is divided into five different levels: PN, AND, BSN, MASTERS, DOCTORATE; capacity is divided into five-level indicators: patient-centered care, interdisciplinary teamwork, evidence-based practice, quality improvement, information, there are 14 secondary indicators[12]. The ability to apply when the American Nurses CNS team tabulation object tagging, develop more targeted nursing skills [13]. Nurses, nursing core competencies developed by the Conference of the Asia Pacific region is

\footnotetext{
* Not:This work was financially supported by the science and technology project(2010-339) of Jilin province; The Jilin Social Science Foundation (2013B313) ; The Education Science Foundation of Jilin Province (GH13096).
} 
divided into three primary indicators of the scale, 11 secondary means, 22 three indicators.

Canada's core competency framework developed in 2010, has four-level indicators, 34 secondary indicators, 31 three indicators ${ }^{14}$. Chinese Liu (2008) the use of international nurse

Chinese Liu (2008) the use of international nurses will develop the ability to frame template two semi-open-ended questions to gather the views of 38 Chinese nursing professionals, and analyzed by content template method[14].

Finally get registered nurse for China's core competency framework, a framework for seven dimensions, 58 entries.

\section{Differences in national indicators of core competence of nurses}

Maine 2013 nurse care team to develop core competencies for the 11-level indicators, relative to the US in 2010 OKLAHOMA scale for a more perfect state, in the past, based on the increased expertise, patient-centered care, evidence-based practice, communication, information and technology, security, geriatrics knowledge, quality improvement which eight projects closer to the core concept of modern nursing.

But reducing the previous research, education coaching skills, ethical decision-making that three projects. I think we can be added to the three indicators of core competencies of nurse projects. Canadian Nurses core competency level indicators small scale, three-level indicators compared with the American Nurses Core Competence Scale: professional roles, responsibilities, quality, health assessment and diagnosis, treatment, management, health promotion and the prevention of damage. Where professional roles, responsibilities include clinical practice, cooperation consulting, research skills, leadership ability.

Similar health assessment and diagnosis and care and information technology, management and treatment of clinical care is similar to health promotion and prevention, injury and quality improvement, safety similar.

Compared with the latest Maine Nurse Core Competence Scale lack of expertise, patient-centered care, geriatrics knowledge. British nurse core competency scale will be divided into nine levels nurse stage, the ability of nurses into knowledge, skills and core knowledge and skills, the core and the special skills of nurses were elaborated personal development, support teams and service development, personal development, he also includes communication, patient development, health and safety, service development, quality, equality diversity six projects.

Compared with the United States more refined ability to index each level nurses, but did not mention the content of evidence-based practice projects, systems practice, IT, geriatrics. I believe that for nurses hierarchical levels can be quantified, help assess the level of nurses. China registered nurses core competency scale is divided into seven dimensions, 58 entries, seven dimensions are: critical thinking research, clinical care, leadership, relationships, legal ethical practice, professional development, educational counseling ${ }^{11}$. Relative to the US scales not patient-centered, evidence based practice, communication, information technology, security, quality improvement, geriatrics content. I think we can add items as appropriate in accordance with national conditions.

\section{Conclusions}

In summary, the core competencies of great significance in the field of nursing profession. Foreign attention and research on core competencies are more nurses are older, and our research and discussion on the core competencies of nurses less. Therefore, we should boldly learn from foreign experience in core competencies, attention and development of nurses, research nurses core competencies in order to improve the overall quality of our nurses, promote the development of the nursing profession.

\section{References}

[1] Riqiang Huang, Yongming Huang. Core skills of a United Kingdom of vocational education's new hot. comparative education research, ,2 (2): 80-82,. 2004. (In Chinese)

[2] Perspectives on Nurse Practitioner/Advanced Practice Nursing in the USA.2010.

[3] Nursing competencies by educational level: Guideline for nursing practice and education in OKLAHOMA .Oklahoma City, OK 73106 (405) 962-1800, 2012.

[4] MA, MSc, RGN, RNT. ICN Framework of Competencies for the Nurse Specialist. by ICN - International Council of Nurses. Fatwa Affara, 1015, 2011.

[5] Ottawa , Canadian Nurses Association. Canadian nurse practitioner core competency framework: Author. 1-28,2010.

[6] Ann Bradshaw PhD, SRN, DipN.Journal of Clinical Nursing, Volume 9, Issue 3, pages 321-329, May 2000.

[7] Clinical nurse specialist core competencies. The national CNS competency task force, 2010.

[8] Maine nurse core competencies. Re-imagine Maine's nursing education and practice, 2013.

[9] Core competences for nursing children and young people. RCN Competences. Royal college of nursing.2011.

[10] CDR Nicole Polinsky, NC, USN Deputy Lead, Clinical Excellence. Navy Nursing Clinical Competencies(2010)

[11] Liu Ming, Yin Lei. The registration nurse core ability evaluation meter structure validity confirmation factor analyzes[J]. China nursing magazine,3( 3): 204-205. 2008.( In Chinese)

[12] Anne C. Thomas, PhD, ANP-BC, Nurse practitioner core competencies. April 2011

[13] Integrated core career and competence framework for registered nurses, Royal college of nursing. June 2012.

[14] Core Competency Framework. Canadian Nurse Practitioner, Canadian nurses association. May2010. 